\title{
Two-flash thresholds as a function of flash luminance and area
}

MARK F. LEWIS 1

CIVIL AEROMEDICAL INSTITUTE, FAA

Two-flash thresholds were obtained from three dark-adapted Ss by means of a two-interval, temporal forced-choice technique involving foveally fixated circular targets varying in luminance and area. The Ss were instructed to report the temporal position of the longer of two pairs of 1-msec flashes, a comparison pair with an interflash interval of $I \mathrm{msec}$, and a test pair with a varying interflash interval. The Ss were informed about the accuracy of their responses after each response. For all three Ss the results replicated a previous finding that two-flash thresholds are a negatively accelerated function of flash luminance, but the function was shown to be dependent on area, a greater threshold change occurring at smaller areas. Two-flash thresholds were also found to be a decreasing function of stimulus area, with the greatest threshold change occurring at low luminances.

Recently there have been several investigations into the effects of flash luminance and duration upon two-flash thresholds. These studies indicate that at low energies (i.e., with short duration, low luminance flashes) two-flash thresholds are a decreasing function of flash luminance (Lewis, 1967); at higher energies (i.e., with flashes of longer duration and higher luminances) two-flash thresholds are independent of luminance (Keitzman, 1967). The current study replicates the low energy luminance effects and extends the problem to include the effects of stimulus area.

\section{Subjects}

\section{METHOD}

Two Ss were an undergraduate man and woman of the University of Oklahoma. An additional S (F.A.) was a woman research technician at the Civil Aeromedical Institute. Only the latter was familiar with the purpose and design of the experiment. All Ss were emmetropes. All Ss were screened for color vision deficiency on a battery of tests that included the A.O.-H.R.R. and the Dvorine plates, the Farnsworth-Munsell 100-hue test, the Farnsworth Dichotomous (Panel D-15) test, and an anomaloscope examination. No evidence of color deficiency was found.

\section{Apparatus}

The apparatus, previously described (Lewis, 1967), included a Maxwellian view optical system (Fig. 1) with a Sylvania Glow Modulator tube used as light source, and associated logic for control of stimulus durations. Luminance was controlled with neutral density filters (Bausch \& Lomb). Luminance was calibrated with an SEI Exposure Photometer using a method described earlier (Lewis, 1965).

\section{Procedure}

The procedure adopted was a variation of the Block Up and Down Two Interval Forced-choice (BUDTIF) method described by Campbell (1963), the method modified for a random double staircase (Cornsweet, 1965). After dark adapting for $10 \mathrm{~min}$, the $\mathrm{S}$ adjusted the intensity of four fixation lines until they were just visible and on an auditory ready signal, pressed a button to start a trial. A low intensity white noise defined two l-sec observation intervals separated by $1 \mathrm{sec}$. A pair of $1-\mathrm{msec}$ flashes was presented in each interval: in one interval a comparison pair with an interflash interval of $1 \mathrm{msec}$ was presented so that the total duration of the comparison stimulus was $3 \mathrm{msec}$; in the other interval a test pair with a variable interflash interval was presented. Onset of test and comparison stimuli followed onset of noise by $40 \mathrm{msec}$. The observation interval in which the test stimulus appeared was randomly varied from trial to trial. The $S$ was instructed to report the temporal position of either the longer or the dimmer stimulus. The $S$ was informed about the accuracy of his responses by a tone that came on momentarily following correct responses.

Within each staircase the stimuli were presented in blocks of eight trials with a minimum intertrial interval of $20 \mathrm{sec}$. The interflash interval for the test stimulus was changed according to the following rules: If the $S$ was right more than six times in a block of eight trials, the interflash interval was decreased by 1 msec. If the $S$ was right less than six times, the interflash interval was increased by $1 \mathrm{msec}$. If the $\mathbf{S}$ was right exactly six times, the test stimulus was unchanged. Two independent staircases were run, one starting at a short interflash interval, the other at a relatively long interval. On any trial the particular staircase which was presented was randomly determined. Twenty blocks of eight trials in each of the two staircases comprised a single session. Each session lasted approximately $120 \mathrm{~min}$. At any fixed luminance and area the staircases were maintained across sufficient sessions for stable estimates of threshold to be achieved; the staircases were maintained across at least three sessions at each condition. The mean interflash interval for the last two sessions at each luminance and area was the threshold. Initially several sessions were required for each $S$ to stabilize at a consistent threshold value. The required number of practice sessions varied from 3 for S R.M. to 18 for $S$ B.J.

The luminances used were $2.0,6.0,20,63$, and $200 \mathrm{~mL}$. Diameters of the circular targets were 5, 10, 20, and 30 min of arc. For each $S$ the order in which luminances and areas were presented was random.

\section{RESULTS AND DISCUSSION}

Figure 2 shows the two-flash thresholds for each $\mathrm{S}$ as a function of flash luminance at each area. Figure 3 shows the same data plotted as a function of area with luminance as the parameter. In both figures the ordinate indicates the total duration of the test stimulus; the vertical bars represent one standard deviation. Figure 4 shows the same data averaged across Ss. The dashed lines connect with the point for which (due to insufficient time) data from S B.J. are not available. Inspection of Figs. 2 and 3 indicates that the data from S B.J. are elevated relative to those of Ss R.M. and F.A.; consequently, the point is probably depressed from the value to be expected were the datum from S B.J. available.

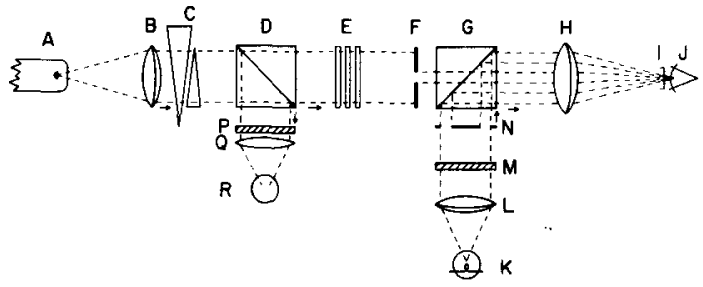

Fig. 1. Optical system used in the present study. Achromatic lens (B) collimates light from a Sylvania glow modulator tube (R1131C), the collimated beam passing through neutral density filters (E), neutral density wedge (C), field stop (F), and beam splitters (D) and (G) before being focused in the plane of the observer's pupil (J), by achromatic lens $(H)$. I is a $2 \mathrm{~mm}$ artificial pupil. Light from an incandescent lamp (K) is collimated by an achromatic lens (L), this collimated beam passing through a red filter (Wratten No. 24) (M), and fixation field stop (N), before being added to the stimulus beam through beam splitter (G). Beam splitter (D) samples a portion of the light from the glow modulator tube passing it through Kodak Wratten filter No. 106 before being focused by lens (Q) on an RCA 1P39 photo multiplier tube used for stimulus monitoring. 

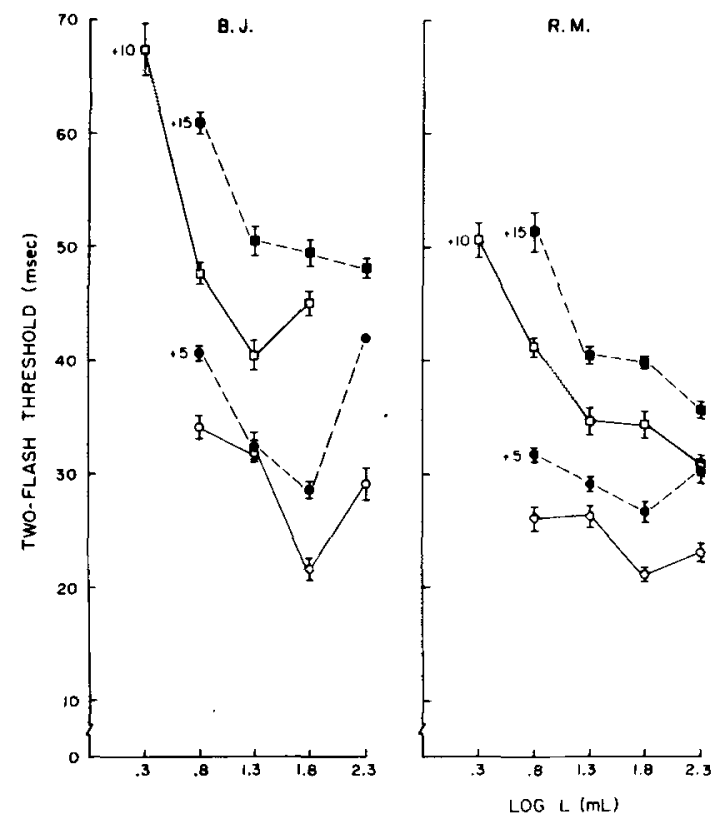

Figures 2 and 4 indicate that two-flash thresholds decrease in a negatively accelerated fashion as luminance increases. At the two larger areas there appears to be a reversal at the highest luminance. This effect was not clearly indicated in the data for all Ss (Fig. 2), nor was a reversal of this kind found in previous studies (Kietzman, 1967; Lewis, 1967). Examination of the data for two of the Ss suggests that the slope of the function decreases as area increases.

Figures 3 and 4 indicate that two-flash thresholds decrease as area increases. This effect is most clearly seen at the lowest luminance. Examination of the data from individual Ss suggests that the effects of area decreases as luminance increases.

In a previous study (Lewis, 1967) it was suggested that the two-flash threshold may be interpreted as estimating the critical duration, $t_{c}$, of Bloch's law. It was also suggested that two-flash thresholds yield direct estimates of the critical duration, unlike

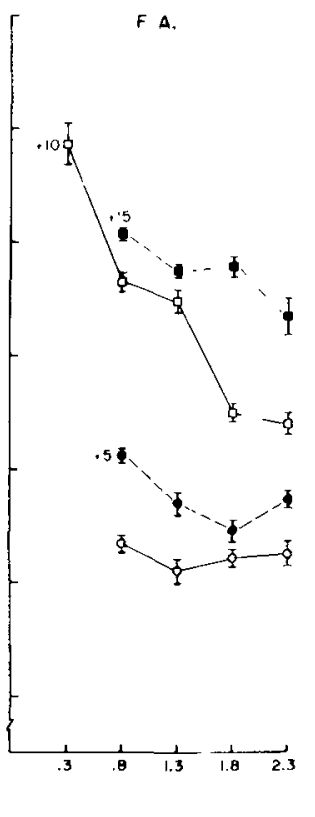

Fig. 2. Two-flash threshold (mec) as a function of $\log$ luminance $(\mathrm{mL})$ for three Ss. Areas are indicated as follows: open circle 30, filled circle 20 , open square 10 , filled square 5 , minutes of arc diameters. Curves have been shifted upward along the ordinate by an amount indicated next to each function. threshold studies which estimate the critical duration from the intersection of the fitted line representing Bloch's law $(\mathrm{Lt}=\mathrm{C}$ ) with the fitted line representing the relation, $L=$ constant. Indeed, it may be that calculating critical duration from such fitted lines overestimates the critical duration, here taken to be the duration at which complete reciprocity ends, by confounding it with what Piéron (1965) has called "utilization time," the duration within which there is only partial integration of energy. With this interpretation the results of the previous study indicate that the critical duration for Bloch's law decreases as a negatively accelerated function of luminance in the dark-adapted eye. That result has been replicated in the current study.

The relation between the critical duration and stimulus area has been the subject of a number of previous investigations. Graham and Margaria (1935) found that as stimulus area increased, there was a less abrupt change from total integration (the relation

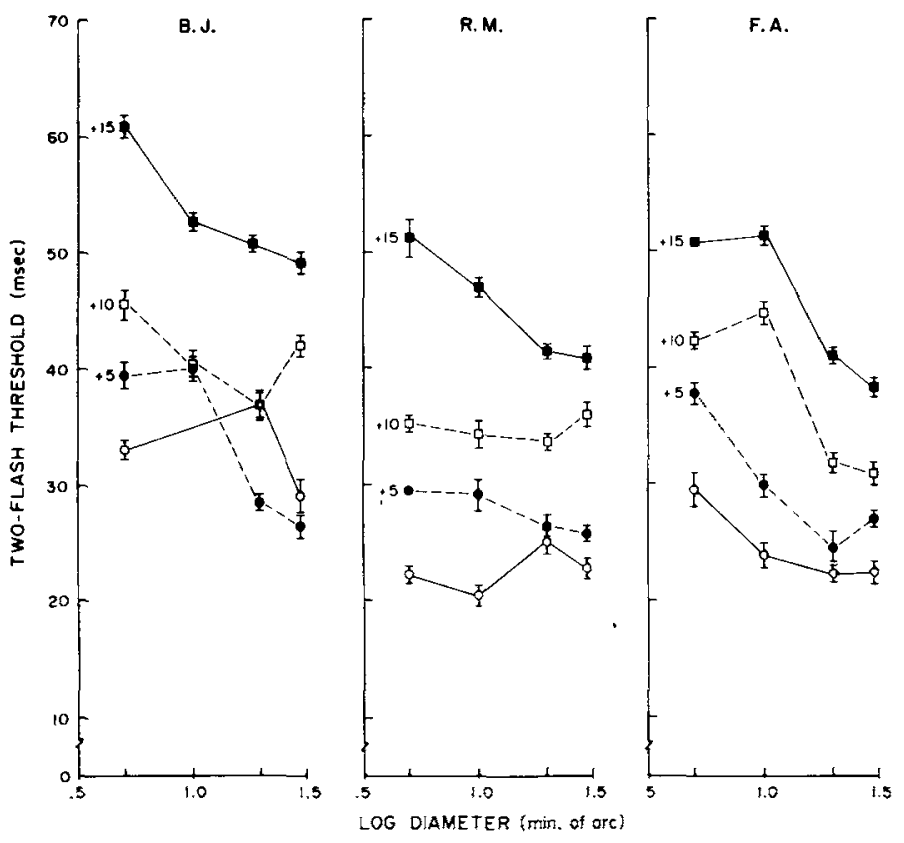

Fig. 3. Two-flash threshold (msec) as a function of the log diameter (in minutes of arc) at each luminance level. Luminances are ind;cated as follows: open circle 2.3 , filled circle 1.8 , open square 1.3 , filled square $.8 \log \mathrm{mL}$. Curves have been shifted upward along the ordinate by amounts indicated next to each function. 


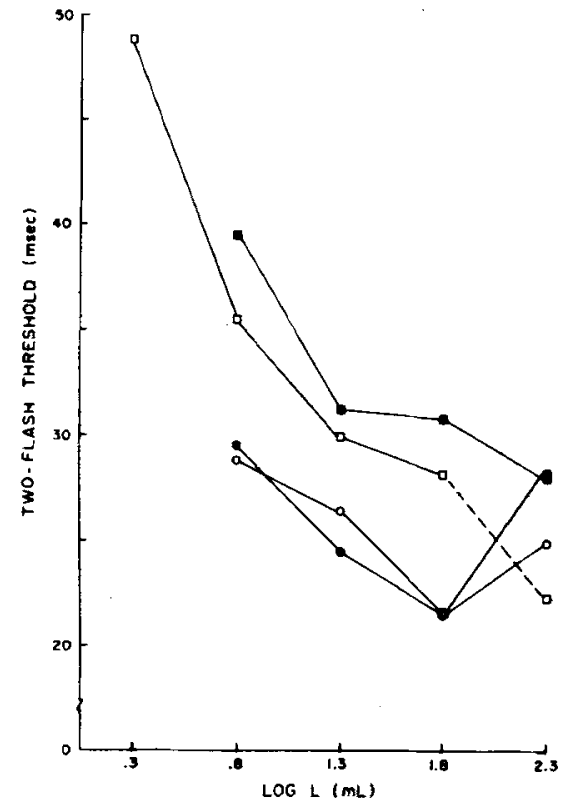

$\mathbf{L t}=\mathrm{C})$ to the relation $\mathbf{L}=$ constant; at a large area the transition was gradual; at the smallest area $(2 \mathrm{~min})$ the transition was abrupt. Moreover the point of departure from strict reciprocity, i.e., the critical duration, decreased as area increased. The same result is obtained by fitting straight lines to their data and taking the intersection as the critical duration. Baumgardt and Hillman (1961) found abrupt changes to occur at approximately $100 \mathrm{msec}$, independent of area, but beyond the critical duration their data show evidence for partial integration occurring up to the longest duration employed $(1 \mathrm{sec})$. The investigation by Sperling and Joliffe (1965) with three stimulus diameters (4.5 min, $45 \mathrm{~min}, \& 3$ deg) indicates departures from complete reciprocity occurred at shorter durations for the larger areas; their data also indicate partial integration occurring at durations well beyond the critical duration. If the critical duration is derived from the Sperling and Joliffe data by following the convention of fitting straight lines representing the relations $\mathrm{LT}=\mathrm{C}$ and $\mathrm{L}=$ constant, the critical
Fig. 4. Two-flash threshold (in msec) as a function of $\log$ luminance (in $\mathrm{mL}$ ) and $\log$ diameter (in minutes of arc). Average of three Ss. On the left stimulus diameters are indicated as follows: open circle 30 , filled circle 20 , open square 10 , filled square $5 \mathrm{~min}$ of arc. On the right luminances are indicated as follows: open circle 2.3 , filled circle 1.8 , open square 1.3 , and filled square $.8 \log \mathrm{mL}$

durations obtained from the intersections of these straight lines do not vary with area, a situation that is in accord with the suggestion made above that such a procedure confounds critical duration with utilization time. If two-flash thresholds may be interpreted as directly estimating the critical duration rather than utilization time, then the data of the current study indicase that critical duration decreases as area increases; this effect is seen most clearly at the lowest luminance used. As the luminance level was increased relative to the level of adaptation, the effect of area became increasingly smaller.

Maneke (1958) has suggested that the two-flash threshold may be discussed as a limiting case for critical flicker-fusion frequency. To facilitate comparisons with flicker data, the data of the current study have been replotted in Fig. 5 to show the reciprocal of the two-flash thresholds as a function of stimulus parameters. Figure 5 indicates that with the two smaller areas critical flicker frequency (as measured with the two-flash threshold) increases with lumi-
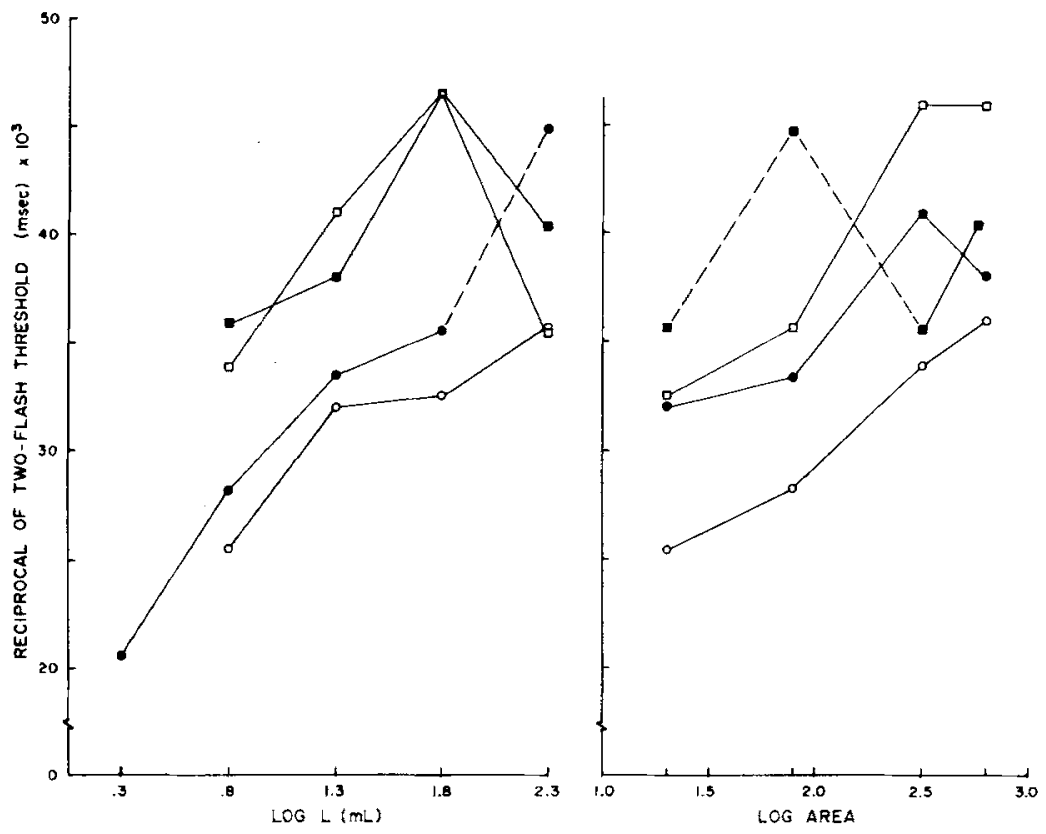

Fig. 5. Reciprocal of two-flash threshold as a function of $\log$ luminance and $\log$ area. On the left stimulus diameters are indicated as open circle 5 , filled circle 10 , open square 20 , filled square 30 min of arc. On the right luminances are indicated as follows: open circle .8 , filled circle 1.3, open square 1.8, filled squares 2.3 $\log \mathrm{mL}$. The reciprocals have been multiplied by an arbitrary constant $\left(10^{3}\right)$ to facilitate comparison with conventional CFF data. 
nance; at the two larger arcas there are significant reversals at the highest luminance investigated.

These data appear to be consistent in form with the results of conventional investigations of $\mathrm{CFF}$ as a function of luminance (Hecht \& Verrijp, 1933). although the slopes of the current functions are greatly reduced. However, in the case of the current data stimulus luminance was varied independently of adaptation level.

Figure 5 indicates that at the lowest uminance level employed, CFF is a linear function of $\log$ area, i.e., these data are in line with the Granit-Harper law. At luminances well above the level of adaptation there are significant departures from the Granit-Harper law such that CFF does not appear to be systematically related to stimulus area. It must be stressed that the data are not strictly comparable with conventional CFF data in that pulse-to-cycle fraction is totally confounded with the dependent variable in the present study, whereas in conventional studies pulse-to-cycle fraction is usually held constant.

\section{REFERENCES}

BAUMGARDT, E., \& HILLMAN, B. Duration and size as determinants of peripheral retinal response. J. Opt. Soc. Amer., 1961, 51, 340-344.

CAMPBELL, R. A. Detection of a noise signal of varying duration. J. Acoust. Soc. Amer., 1963, 35, 1732-1737.
CORNSWEET, T. N. The staircase-method in psychuphysics. Amer. J. Psychol., 1965, 75, 485-491.

GRAHAM, C. H., \& MARGARIA, R. Area and the intensity-time relation in the peripheral retina: Amer. J. Physiol, 1935, 113, 299.305.

HECHT, S., \& VERRIJP, C. D. Intermittent stimulation by light. III. and IV. J. gen Physiol., 1933, 17, 251-186.

KIETZMAN, M. L. Two-pulse measures of temporal resolution as a function of stimulus energy. J. Opt. Soc. Amer., 1967, 57, 809.813.

LEWIS, M. F. Category judgments as functions of flash luminance and duration. J. Opt. Soc. Amer., 1965, 55, 1655-1660.

LEWIS, M. F. Two-flash thresholds as a function of luminance in the dark-adapted eye. J. Opt. Soc. Amer., 1967, 57, 814-815.

MAHNEKE, A. Fusion thresholds of the human eye as measured with two or several light flashes. Acta Ophthalmol., 1958, 36, 12-18.

PIÉRON, H. Vision in intermittent light. In W. D. Neff (Ed.), Contributions to sensory physiology, Vol. L. New York: Academic Press, 1965, 179-264.

SPERLING, H. G., \& JOLIFFE, C. L. Intensity-time relationship at threshold for spectral stimuli in human vision. J. Opt. Soc. Amer., 1965, 55, 191-199.

NOTE

1. Address: Psychology Laboratory, Aeromedical Research Branch, Civil Aeromedical Institute, P. O. Box 25082, Oklahoma City, Oklahoma 73125.

(Accepted for publication July 19, 1968.) 